\title{
Optimizing the Raman signal for characterizing organic samples: The effect of slit aperture and exposure time
}

\author{
João Carlos Lázaro a , Marcos Tadeu T. Pacheco ${ }^{\mathrm{b}}$, Kátia Calligaris Rodrigues ${ }^{\mathrm{a}}$, \\ Carlos José de Lima ${ }^{b}$, Leonardo Marmo Moreira ${ }^{b, *}$, Antonio Balbin Villaverde ${ }^{b}$ and \\ Landulfo Silveira Jr. ${ }^{\text {b }}$ \\ ${ }^{a}$ Instituto de Pesquisa e Desenvolvimento, Universidade do Vale do Paraíba, São José dos Campos, SP, \\ Brazil \\ ${ }^{\mathrm{b}}$ Núcleo de Engenharia Biomédica, Universidade Camilo Castelo Branco, São José dos Campos, \\ SP, Brazil
}

\begin{abstract}
The present work is focused on the influence of the slit aperture and time exposure of the infrared light on the Charge Coupled Device (CCD) in relation to their physical effects, in order to improve the Raman spectrum characteristics. Indeed, the alterations in slit aperture and CCD time exposure affect significantly important spectral properties, such as the spectral intensity, Signal to Noise Ratio (SNR) and band width resolution of the Raman spectra. Therefore, the present proposal has the aim of to found the optimum conditions of instrumental arrangement, involving the minimum collection time and maximum signal quality in dispersive Raman spectrometers. Samples of dehydrated human teeth and naphthalene were evaluated with a Raman dispersive spectrometer employing excitation wavelength of $830 \mathrm{~nm}$ in several integration times and spectrometer slit apertures. The analysis of the spectral intensity, SNR and band width of selected Raman peaks allowed to infer that these properties of a dispersive Raman spectrum depend directly of the exposure time on the detector as well as spectrograph slit aperture. It is important to register that the higher SNR was obtained with higher exposure time intervals. To the samples evaluated in the present article, the band width has lower values for slit apertures of 100-150 $\mu \mathrm{m}$, i.e., in this aperture range the spectral resolution is maximum. On the other hand, the intensity and SNR of the Raman spectra becomes optimal for slit apertures of 150-200 $\mu \mathrm{m}$, since this aperture does not affect significantly the integrity of the Raman signal. In this way, we can to propose that in approximately $150 \mu \mathrm{m}$, it is possible to obtain an optimum condition, involving spectral resolution as well as SNR and spectral intensity. In any case, depending of the priorities of each spectral measurement, the instrumental conditions can be altered according with the necessities of each specific chemical analysis involving a determined sample. The present data are discussed in details in agreement with recent data from literature.
\end{abstract}

Keywords: Raman spectroscopy, spectral resolution, band width resolution, signal to noise ratio (SNR)

\section{Introduction}

Dispersive Raman spectroscopy has become very popular in the last decades as a powerful tool for biochemical analysis and diagnosis, involving various biological tissues and molecules of biochemical interest. In fact, this spectroscopic technique allows the identification as well as qualitative and quantitative analysis of various biological indicators, which present characteristic Raman peaks, that

\footnotetext{
${ }^{*}$ Corresponding author: Leonardo Marmo Moreira, Instituto de Pesquisa e Desenvolvimento, Universidade do Vale do Paraíba, Av. Shishima Hifumi, 2911, 12244-000, São José dos Campos, SP, Brazil. E-mail: leonardomarmo@gmail.com.
} 
work as "fingerprint" of each sample. This employment of Raman spectroscopy has been done in order to elucidate the physico-chemical mechanisms of several biological processes, such as physiological mechanisms, development of several diseases, and quantification of biomolecules of great biochemical relevance. Furthermore, the clinical applications of this spectroscopy, using "in vivo" analysis to obtain a precise diagnosis, demand a minimally invasive procedure of short duration, which allows higher quality of life of patients with several grave diseases, such as cancer and atherosclerosis. Thus, it has been developed various efforts aiming to decrease the time of analysis to avoid procedures of higher stress to the patients [1-4].

The Raman spectroscopy is a vibrational technique based in a physical process called Raman scattering, that is an inelastic phenomenon that occurs when a photon from a light interacts with a molecule. In this process, energy can be transferred from the light to the molecule, implying that the scattered photon presents loss of energy in relation to the excitation radiation, i.e., the scattered photon has a higher wavelength when compared with the incident light, characterizing the called Stokes shift. On the other hand, it is also possible, but with lower probability, the occurrence of a gain of energy of the scattered photon. This occurs as function of the Distribution of Boltzmann to the vibrational levels, which can present a small number of molecular species in the excited state. In this way, this excited molecules could be promoted to a second level of excitation and, with the relaxation process, would emit a photon with higher energy than the excitation radiation, characterizing the process named Anti-Stokes shift.

The difference among the incident photon energy and the re-emitted (scattered) one depends on the vibrational energy levels from the sample [5]. Therefore, this physical process of Raman scattering results in the emission of photons in a particular wavelength, which leads to formation of characteristic spectral peaks with specific intensity and band width, depending on the laser power, slit aperture and exposure time of the irradiated sample [6-8]. In fact, Fig. 1 demonstrates the relationship between "capability of spectral resolution" $\Delta_{\mathrm{CRE}}$ and light power transmitted through the aperture of slit [6].

An adequate process of Raman signal determination leads to a reliable identification of spectral wavelength, minimum band width (high resolution), high spectral intensity (high sensitivity) and high SNR. In this way, the crescent application of dispersive Raman spectroscopy in life sciences has motivated a great

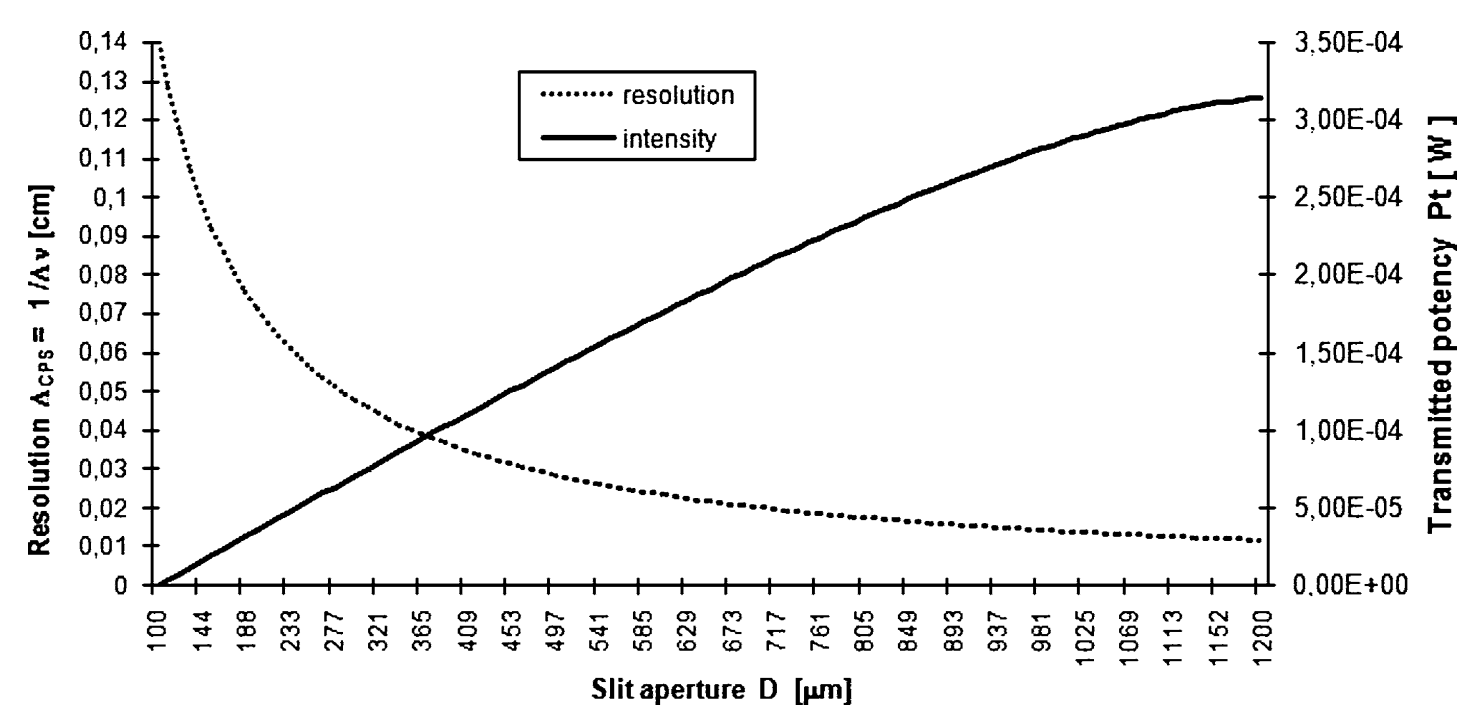

Fig. 1. Relationship between "capability of spectral resolution" $\Delta_{\mathrm{CRE}}$ and light power transmitted through the aperture of slit. 
effort by several research groups in order to improve the instrumental conditions of Raman signal analysis [9]. Furthermore, the identification of the principal biological molecules in each biochemical sample analyzed by this technique and the subsequent differentiation between healthy and diseased tissues has been developed [10,11].

The improvement of the signal to noise ratio (SNR) in Raman spectra is usually obtained by the increase of the laser power up to a determined level below the thermal damage threshold. Currently, in studies focused on biological samples, Raman spectrometers employ infrared excitation, since this radiation reduces significantly the organic sample auto fluorescence, permitting that the Raman photons scattered by the sample can be more easily detected [5,12]. On the other hand, the intensity of scattered light decreases proportional to $\lambda^{-4}$ [13], reducing significantly the amount of scattered photons available. The optical window between 700 and $1000 \mathrm{~nm}$ is the best choice for Raman excitation in organic samples. Near-wavelength infrared (ranging from 1.4 to $3 \mu \mathrm{m}$ ) cannot be used in dispersive instruments due to lack of sensitivity in silicon-based CCD cameras [14]. At present time, some researchers have presented the CCD based on germanium, which could bring the fluorescence-free Raman spectroscopy to the short infrared spectrum [15].

Increasing the time exposure is another possibility for a better SNR. But several biological applications need a fast time between exposure and collection, due to sample degradation, moving, decanting, in vivo clinical applications [16,17]. For so, the collection time should be set to the minimum for an optimal signal quality (band intensity and bandwidth). Nevertheless, the slit aperture from the spectrograph, that controls the spectral resolution, should be set to a value that permits higher light throughput with minimal spectral distortion [6-8]. Actually, the slit aperture is the most important parameter for spectral resolution, together with the correct spectrograph-to-CCD focus alignment.

This work proposes to study the influence of slit aperture and detector time exposure in the Raman signal to noise ratio (SNR), band intensity and width parameters in a dispersive Raman instrument, parameters that will define the Raman spectrum quality. For this purpose, two organic samples were used (naphtalene and dehydrated human teeth). Results will indicate an optimal slit aperture and time exposure for analysis of highly scattered samples in a routine Raman experiment.

\section{Material and methods}

The spectrograph used in the experiment is the 250IS (Chromex, Inc., NM, USA) with diffraction grating of 600 lines $/ \mathrm{mm}$, f\#4, in the range of 400-1500 nm, blazed at about $900 \mathrm{~nm}$ [18]. The reciprocal linear dispersion (RLD) for this grating is presented at the spectrograph manual and has a value of about $5.0 \mathrm{~nm} / \mathrm{mm}$. The grating position is step-motor controlled by a hand-held controller. The slit aperture is also hand-held controlled, from $10 \mu \mathrm{m}$ to $2.0 \mathrm{~mm}$ in $10 \mu \mathrm{m}$ steps.

For excitation of Raman signal it was used a semiconductor AsGaAl laser (Micro Laser Systems Inc., Model L4830S, CA, USA) with $100 \mathrm{~mW}$ power and $830 \mathrm{~nm}$ excitation wavelength. The laser was directed to the sample following the scheme described in Silveira Jr. et al. [18]. After reaching the sample, the scattered light was collected by a set of lenses and couples to the spectrograph slit, first passing through a notch filter at $830 \mathrm{~nm}$ before the entrance slit (Spectral Iridian Technologies, model PN-ZX 000080, ON, Canada) to reject the scattered light from the laser. The signal dispersed by the spectrograph was detected by a $1024 \times 256 \mathrm{CCD}$ camera, liquid nitrogen cooled (Princeton Instruments, model LN/CCD-1024-EHR1, NJ, USA), connected to a CCD controller (Princeton Instruments, model ST130, NJ, USA) and a PC for the acquisition and storage of spectra. 
Raman spectra collection were done by changing the optical aperture of the entrance slit from 50 to $400 \mu \mathrm{m}$ and changing the integrating time from $50 \mathrm{~ms}$ to $50 \mathrm{~s}$. The Raman signal was obtained from two organic samples: a naphtalene sphere and a dehydrated human molar tooth. For the human tooth, the spectrum was taken in the enamel portion of the vestibular face.

All spectra were recorded in binary format and converted to ASCII format for post-processing. The Raman wavenumber shift calibration was done by fitting a third-order polynomial of the pixel position with relation to the known naphtalene bands. A fluorescence removal procedure, based on a second derivative algorithm, was applied to all spectra.

Spectra were plotted using Microsoft Excel spreadsheet and band positions, intensities and FWHM (Full Width at Half Maximum) band widths were obtained and tabled. Scatter plot graphics of band widths and band intensities versus slit widths were done depending on the CCD time exposure.

\section{Results and discussion}

The minimum spectral linewidth (SLW) that the dispersive spectrograph could resolve is defined by the expression [19]:

$$
\mathrm{SLW}(\mathrm{nm})=\operatorname{RLD}(\mathrm{nm} / \mathrm{mm}) \times \operatorname{SWD}(\mathrm{mm}) .
$$

In which RLD is the reciprocal linear dispersion of the grating and the SWD is the slit width, for a specific wavelength. The maximum wavenumber resolution (WNR) depends on the scattered wavelength by the expression:

$$
\operatorname{WNR}\left(\mathrm{cm}^{-1}\right)=(\operatorname{SLW}(\mathrm{nm}) / \mathrm{WL}(\mathrm{nm})) \times\left(10^{7}(\mathrm{~nm} / \mathrm{cm}) / \mathrm{WL}(\mathrm{nm})\right) .
$$

In which WL is the wavelength where the resolution is being calculated. Figure 2 shows a plot of the maximum WNR in the range of $850-1000 \mathrm{~nm}$, which is the wavelength range covered by the wavenumber range of most significant Raman bands of biological samples $\left(800-1800 \mathrm{~cm}^{-1}\right)$.

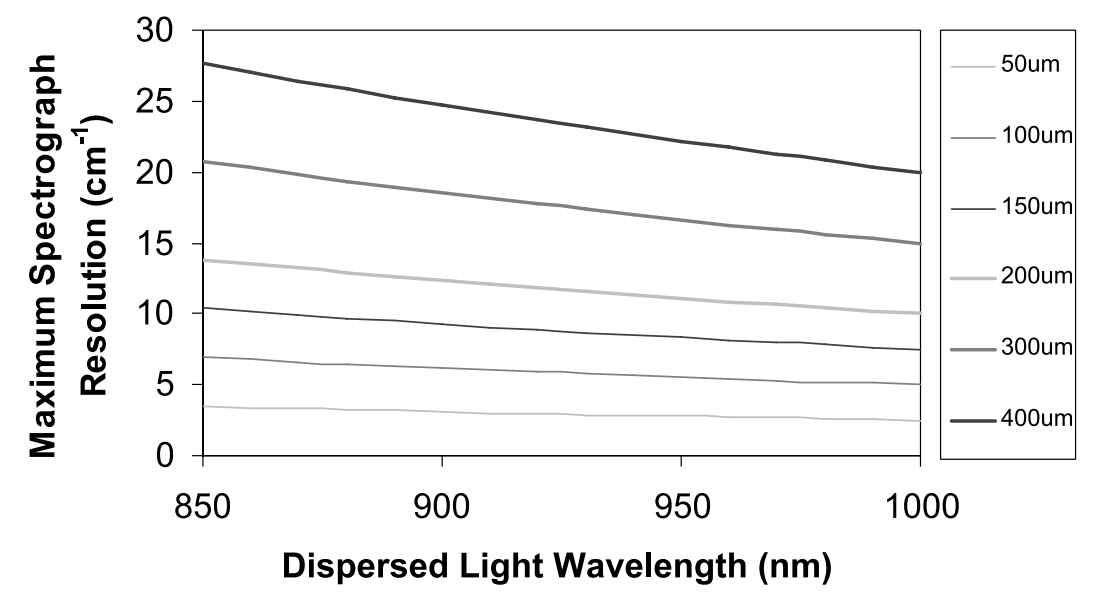

Fig. 2. Plot of the maximum wavenumber resolution (WNR) versus scattered wavelength dispersed by the spectrograph for different slit apertures $(\mu \mathrm{m})$ in the wavelength range commonly used in dispersive Raman spectra of biological samples. 
The WNR for a specific Raman wavenumber should be lower than the most narrow band of the spectrum of interest so the desired band can be read and identified without deformation. Raman bands of biological molecules have relatively broad bands when compared to crystals and polymers, due to the overlap of vibrational bands for most biochemicals presented in cells and tissues [20]. For instance, the calcium hydroxiapatite found in human atherosclerotic plaque has a Raman band position at $960 \mathrm{~cm}^{-1}$ and bandwidth of about $25 \mathrm{~cm}^{-1}$ when obtained in near-infrared, dispersive Raman instruments [21]. Therefore, the instrument should be able to resolve this biological sample Raman band with this minimal resolution. This issue will be analyzed in the band width results.

For spectral analysis, it was used samples of naphtalene and human tooth. Figure 3 shows the Raman spectra of naphtalene and human tooth in vitro with slit of $200 \mu \mathrm{m}$ and time exposure of $50 \mathrm{~s}$. The most intense Raman band of tooth located at $962 \mathrm{~cm}^{-1}$ is attributed to $\mathrm{P}=\mathrm{O}$ stretching mode from calcium hydroxyapatite from enamel [22]. For naphtalene, the most intense Raman band is attributed to mode of vibration at $1382 \mathrm{~cm}^{-1}$ [17]. Due to the positions of such bands that coincide with most important ones found in biological samples (region between 800 and $1700 \mathrm{~cm}^{-1}$ ), they were used to obtain a correlation of exposure time and slit aperture to the signal to noise ratio without loss of spectral resolution.

Figure 4 plot the intensity of the 962 and $1382 \mathrm{~cm}^{-1}$ Raman bands of calcium hydroxiapatite and naphtalene, respectively, as a function of slit width taken with different time exposures. It can be observed that both peak intensities increase following CCD time exposure, with all curves parallel to each other. Also, as slit aperture increases, considering the same integration time, the result is a band intensity increasing for slit apertures of 50,100 and $150 \mu \mathrm{m}$ and at about $200 \mu \mathrm{m}$ the band intensity becomes constant.

Figure 5 plots the SNR, calculated by dividing the intensity of the selected band by the standard deviation of a selected spectral region in the final portion of the spectrum (near $1800 \mathrm{~cm}^{-1}$ ), where there is no Raman band, only spectral noise. It can be observed that the SNR varies depending on the time

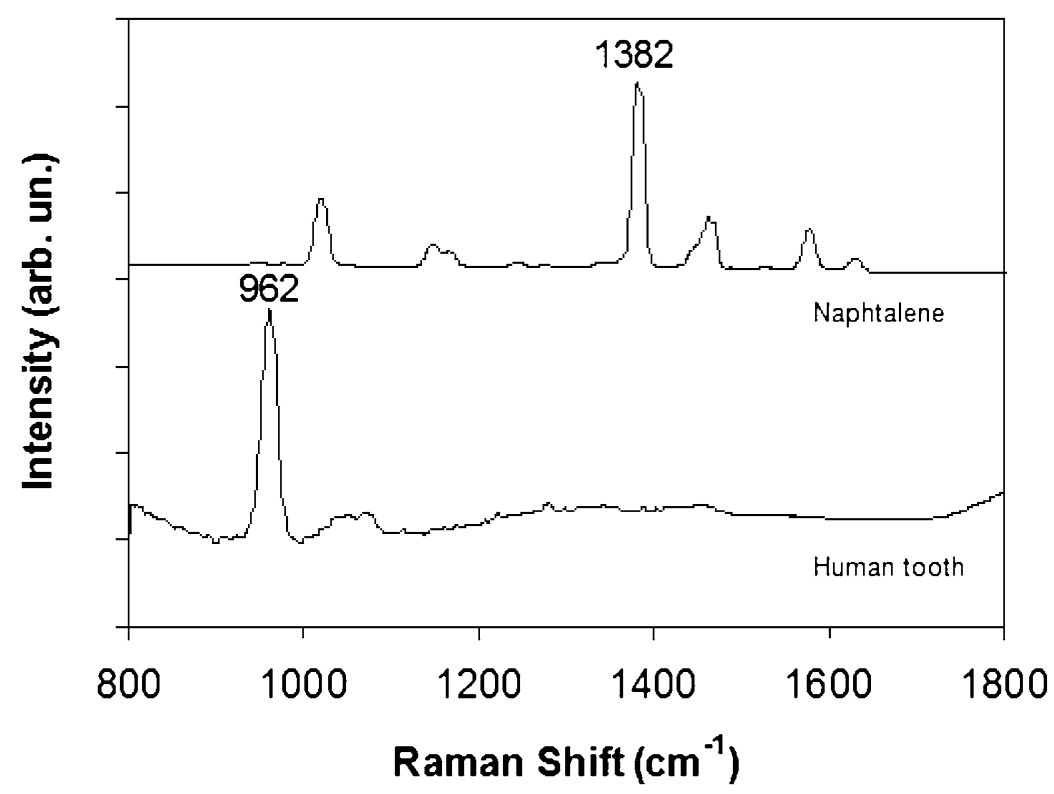

Fig. 3. Raman spectra of naphtalene and human tooth enamel in vitro obtained with $830 \mathrm{~nm}$ excitation, $60 \mathrm{~mW}$ laser power, $200 \mu \mathrm{m}$ slit and $50 \mathrm{~s}$ exposure time. 

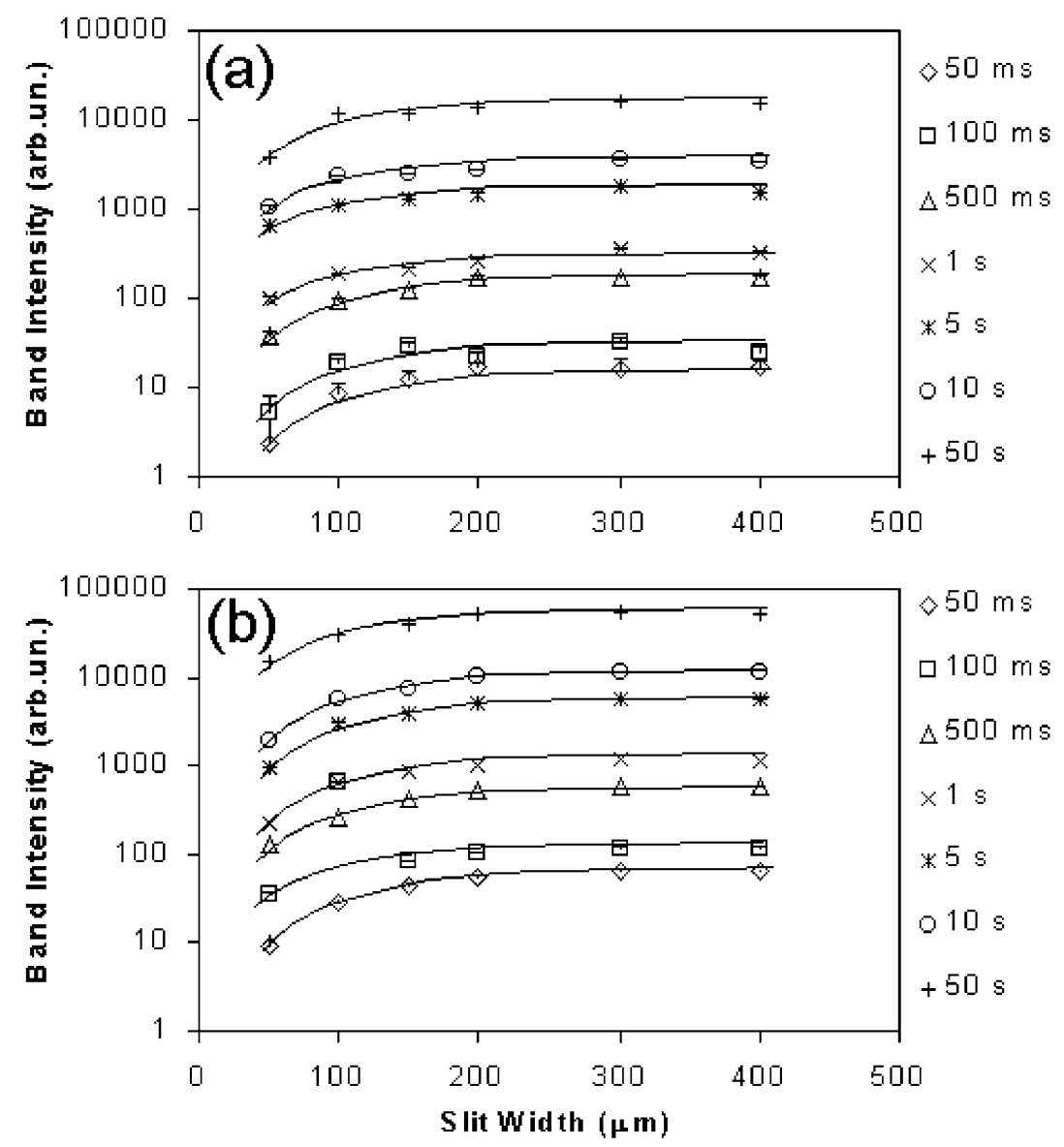

Fig. 4. Plot of the Raman band intensity as a function of entrance slit width for (a) the $962 \mathrm{~cm}^{-1}$ calcium hydroxiapatite band from human tooth, and (b) the $1382 \mathrm{~cm}^{-1}$ naphtalene band taken with different CCD time exposures.

exposure and slit width as well. In general, the SNR increases with time exposure for small slit apertures. However, for larger slits, the SNR keeps constant or even decreases, as, for example, the $962 \mathrm{~cm}^{-1}$ band at small time exposures. The SNR dependence from the slit aperture leads to a statement that good SNR can be obtained with small slit aperture and long time exposure. In general, the SNR decreases for the 300 and $400 \mu \mathrm{m}$, independently of the time exposure. This shows that better SNR is obtained with smaller apertures and higher exposure times. There is not much difference in the SNR for the $962 \mathrm{~cm}^{-1}$ band at slits higher than $300 \mu \mathrm{m}$ in the time exposures of 5, 10 and 50 seconds, indicating that the SNR dependence is only due to the slit aperture, not integrating time.

Figure 6 plot the width of the 962 and $1382 \mathrm{~cm}^{-1}$ Raman bands of calcium hydroxiapatite and naphtalene, respectively, as a function of slit width taken with different time exposures. In spite of the higher scattering of the data for lower slit apertures, it can be seen that the band width follows a 3rd-order polynomial fitting (black line). An interesting result is that, for slit apertures of $50 \mu \mathrm{m}$, the band width become broader than the one found in $100 \mu \mathrm{m}$ for the calcium peak. The lowest band width for calcium hydroxiapatite was found at $50 \mu \mathrm{m}$ with about $17 \mathrm{~cm}^{-1}$ and for naphthalene at $100 \mu \mathrm{m}$ with about $12 \mathrm{~cm}^{-1}$. For slit apertures higher than $150 \mu \mathrm{m}$, the band width increases linearly up to $35 \mathrm{~cm}^{-1}$ for both samples. These results are relatively independent of the time exposure. These results corroborate with 

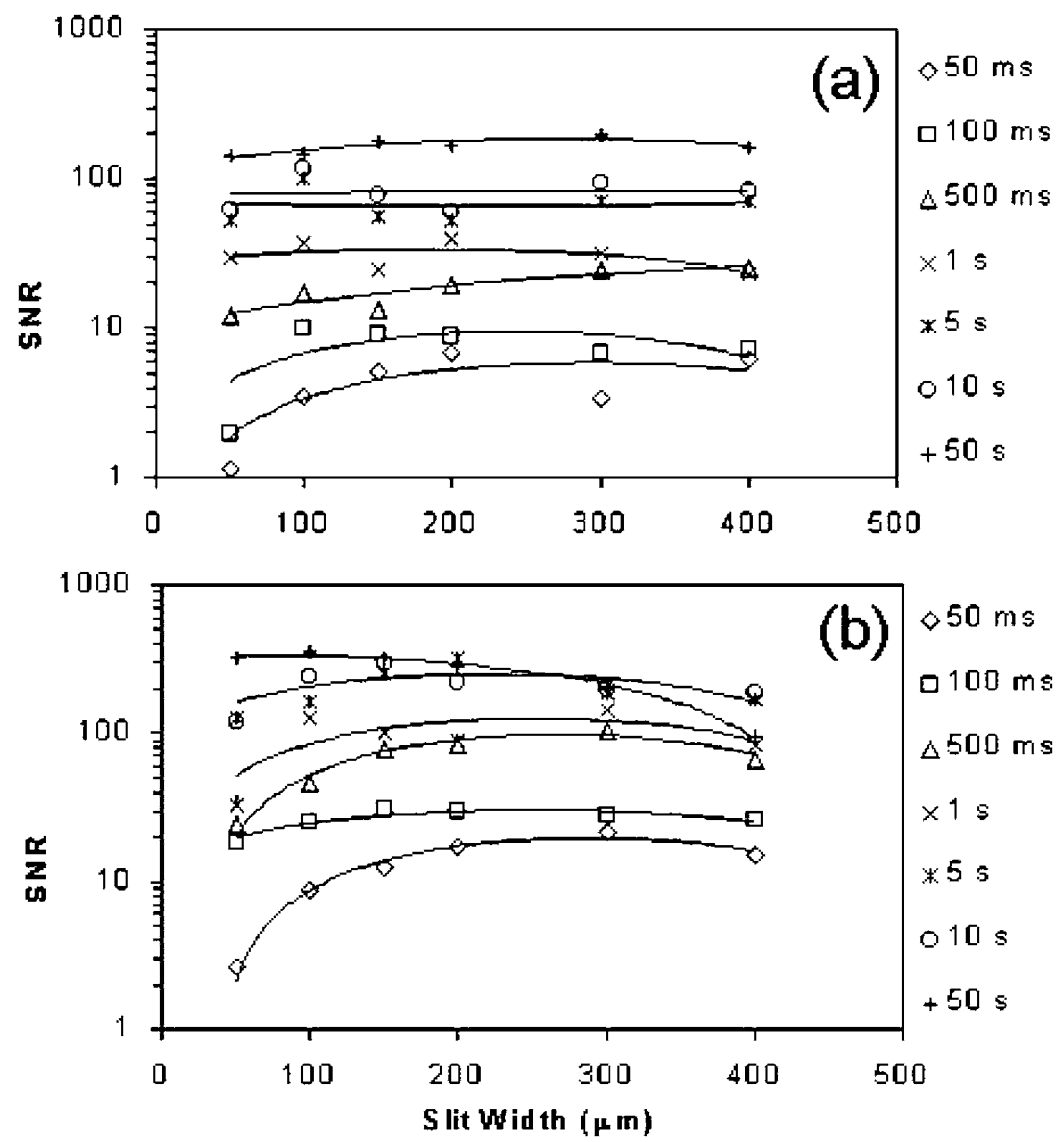

Fig. 5. Plot of the Raman band signal to noise ratio (SNR) as a function of entrance slit width for (a) the $962 \mathrm{~cm}^{-1}$ calcium hydroxiapatite band from human tooth, and (b) the $1382 \mathrm{~cm}^{-1}$ naphtalene band taken with different CCD time exposures.

the one found in Fig. 2, indicating that the resolution becomes worse as the slit aperture becomes high. And also, Fig. 6 shows that the band at $962 \mathrm{~cm}^{-1}$ has higher band width than the band at $1382 \mathrm{~cm}^{-1}$.

Higher spectral resolution is desired to spectrally resolve bands that are closer and could be misidentified by a spectral algorithm. Mainly in the region of $1000-1700 \mathrm{~cm}^{-1}$, where it is found several Raman bands of biomolecules (mainly fatty acids and proteins), a good resolution is found when is used a slit aperture of 100-150 $\mu \mathrm{m}$. Especially it was found the $150 \mu \mathrm{m}$, where a measured band width of about $15 \mathrm{~cm}^{-1}$ for lower Raman shift and $20 \mathrm{~cm}^{-1}$ for higher Raman shift indicates the possibility of identifying bands closer than these band widths.

In terms of spectra intensity, higher time exposures provide a better intensity and SNR. But the curves showed that such higher intensity, for high time exposure (above $1 \mathrm{~s}$ ) is found when slit is set to lower values, from 50 to $150 \mu \mathrm{m}$. For low time exposure (below $1 \mathrm{~s}$ ) the better slit is found from 150 to $200 \mu \mathrm{m}$. These results combined show that slit of $150 \mu \mathrm{m}$ is the better choice for both short and long exposure time to obtain a better SNR.

The possibility of optimization of the width, intensity and SNR of Raman bands by using a slit aperture of $150 \mu \mathrm{m}$ brings benefits to the data analysis. If band width becomes broader, it is more difficult to separate between two closer bands due to the increase of the detected band width. In fact, the determination of stability, resolution, and precision of a Raman spectrometer has been an important focus of 

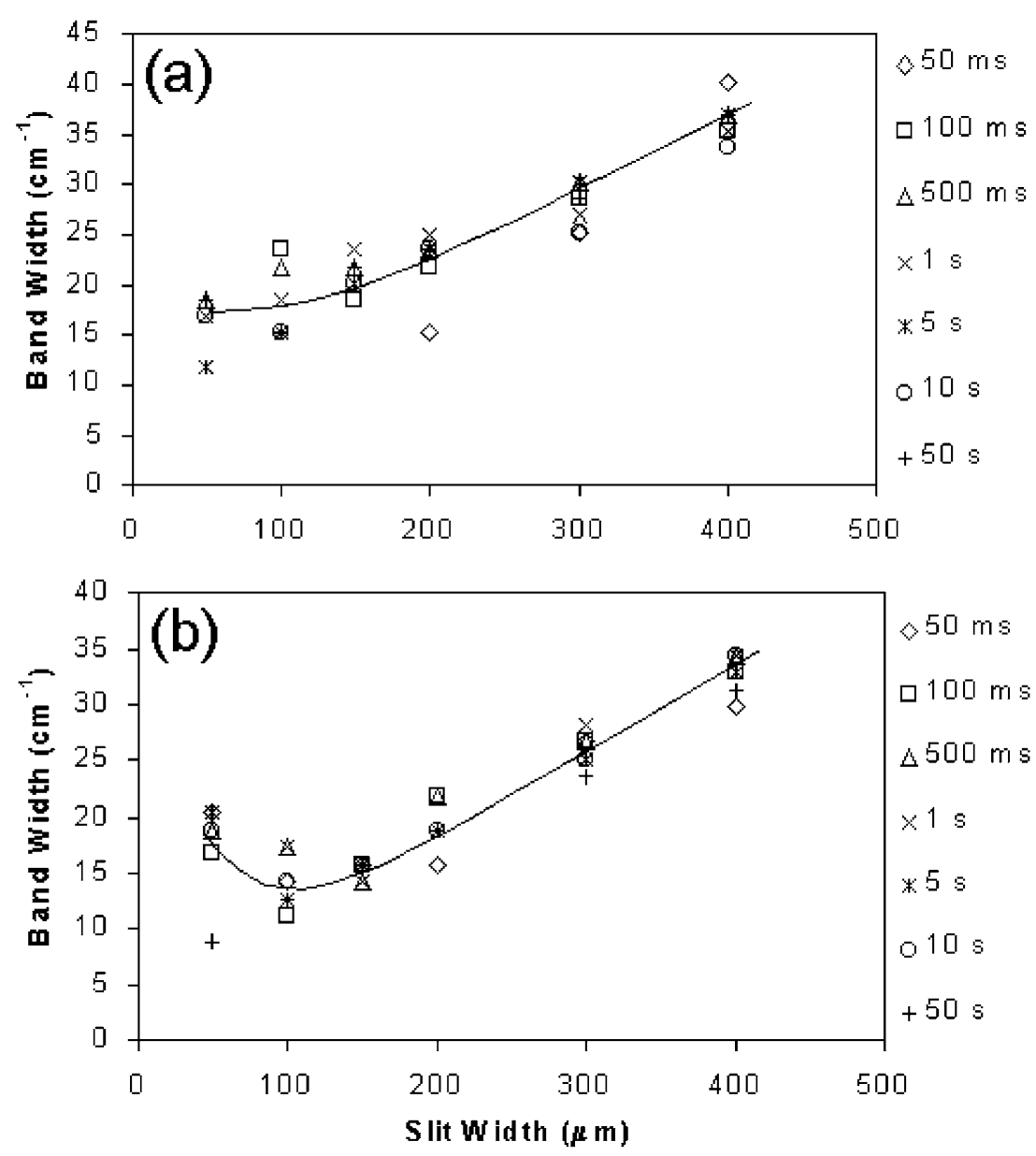

Fig. 6. Plot of the Raman band width as a function of entrance slit width for (a) the $962 \mathrm{~cm}^{-1}$ calcium hydroxiapatite band from human tooth, and (b) the $1382 \mathrm{~cm}^{-1}$ naphtalene band taken with different CCD time exposures.

study involving several groups [23]. When slit becomes thinner, bad SNR is obtained. By setting the correct slit and time exposure, dispersive Raman spectrometer could be able to identify the most weak and narrow band in the whole spectral region without errors in the band identification.

Recently, several studies have been focused on the improvement of the SNR in Raman spectra [24-27]. These efforts are justified as function of the excellent possibilities of analytical applications of Raman spectroscopy, mainly in biomedical purposes. However, some developments are not easily accessible to great number of laboratories that use Raman spectroscopy. In this way, a more simple methodology, which would present less cost and more operational availability, could be very interesting in order to become more widespread the employment of Raman spectroscopy. In fact, spectral properties as high resolution and sensitivity are decisive to determine the success of a specific spectral study, especially in complex evaluations, such as that from biological samples. In this kind of sample the coexistence of several organic compounds with near bands is a constant phenomenon, precluding, in various cases, the precise identification of "fingerprint bands". In this context, the optimization of slit aperture and exposure time are very suitable tools to improve these analysis, since that this methodology does not require use of additional instrumental devices. 


\section{Conclusion}

In the present work, it was found that the band width and SNR of a dispersive Raman spectrum depend on the detector time exposure and spectrograph slit aperture. In fact, the band width has lower values for slit apertures of 100-150 $\mu \mathrm{m}$. Also, the intensity and SNR of the Raman band becomes optimal for slit apertures of 150-200 $\mu \mathrm{m}$. Higher SNR is obtained with high integrating time. Then, the optimal slit aperture to cover a wide range of applications could be set to $150 \mu \mathrm{m}$ in order to optimize the resolution independently of the time exposure.

\section{References}

[1] L.M. Moreira, L. Silveira Jr., F.V. Santos, J.P. Lyon, R. Rocha, R.A. Zângaro, A.B. Villaverde and M.T.T. Pacheco, Raman spectroscopy: A powerful technique for biochemical analysis and diagnosis, Spectroscopy 22 (2008), 1-19.

[2] C.M. Krishna, N.B. Prathima, R. Malini, B.M. Vadhiraja, R.A. Bhatt, D.J. Fernandes, P. Kushtagi, M.S. Vidyasagar and V.B. Kartha, Raman spectroscopy studies for diagnosis of cancers in human uterine cervix, Vibrat. Spectrosc. 41 (2006), 136-141.

[3] D. Rohleder, G. Kocherscheidt, K. Gerber, W. Kiefer, W. Köhler, J. Möcks and W. Petrich, Comparison of mid-infrared and Raman spectroscopy in the quantitative analysis of serum, J. Biomed. Opt. 10 (2005), 031108.

[4] R.A. Shaw and H.H. Mantsch, Vibrational biospectroscopy: from plants to animals to humans. A historical perspective, J. Mol. Struct. 480/481 (1999), 1-13.

[5] E.B. Hanlon, R. Manoharan, T.W. Koo, K.E. Shafer, J.T. Motz, M. Fitzmaurice, J.R. Kramer, I. Itzkan, R.R. Dasari and M.S. Feld, Prospects for in vivo Raman spectroscopy, Phys. Med. Biol. 45 (2000), R1-R59.

[6] J.C. Lázaro, C.J. De Lima, L.M. Moreira, L. Silveira Jr., N.J.F. da Silveira, A.B. Villaverde and M.T.T. Pacheco, Analysis of the alteration in the optical configuration of Raman spectrometer: Optimization of Signal to Noise Ratio (SNR) in a specific wavelength range of clinical interest, Spectroscopy 22 (2008), 467-474.

[7] J.T. Motz, S.J. Gandhi, O.R. Scepanovic, A.S. Haka, J.R. Kramer, R.R. Dasari and M.S. Feld, Real-time Raman system for in vivo disease diagnosis, J. Biomed. Opt. 10 (2005), 031113.

[8] T.C. Bakker Schut, R. Wolthuis, P.J. Caspers and G.J. Puppels, Real time characterization on the basis of in vivo Raman spectra, J. Raman Spectrosc. 33 (2002), 580-585.

[9] C.J. De Lima, S. Sathaiah, L. Silveira Jr., R.A. Zângaro and M.T.T. Pacheco, Development of catheters with low background signal for Raman spectroscopy diagnosis applications, Artif. Organs 24 (2000), 231-234.

[10] A. Mahadevan-Jansen and R. Richards-Kortum, Raman spectroscopy for the detection of cancers and precancers, J. Biomed. Opt. 1 (1996), 31-37.

[11] H.P. Bushman, J.T. Motz, G. Deinum, T.J. Römer, M. Fitzmaurice, J.R. Kramer, A. van der Laarse, A.V. Bruschke and M.S. Feld, Diagnosis of human coronary atherosclerosis by morphology-based Raman spectroscopy, Cardiovasc. Pathol. 10 (2001), 59-68.

[12] K. Tanaka, M.T.T. Pacheco, J.F. Brennan, I. Itzkan, A.J. Berger, R.R. Dasari and M.S. Feld, Compound parabolic concentrator probe for efficient light collection in spectroscopy of biological tissue, Appl. Opt. 35 (1996), 758-763.

[13] J.R. Ferraro and K. Nakamoto, Introductory Raman Spectroscopy, Academic Press, New York, NY, 1994.

[14] K.P.J. Williams and D.L. Gerrard, The use of 752.5 and $799.3 \mathrm{~nm}$ laser excitation in Raman spectroscopy, Opt. Laser Technol. 17 (1985), 245-248.

[15] T. Hirschfeld and B. Chase, FT-Raman spectroscopy: Development and justification, Appl. Spectrosc. 40 (1986), $133-137$.

[16] C.J. De Lima, S. Sathaiah, M.T.T. Pacheco, R.A. Zângaro and R. Manoharan, Side-viewing fiberoptic catheter for biospectroscopy applications, Laser Med. Sci. 19 (2004), 15-20.

[17] C.J. De Lima, M. Simões, L. Silveira Jr., A.B. Villaverde and M.T.T. Pacheco, Optical fiber catheter with distal end bending mechanism control for Raman biospectroscopy applications, Instrum. Sci. Technol. 74 (2008), 107-114.

[18] L. Silveira Jr., S. Sathaiah, R.A. Zângaro, M.T.T. Pacheco, M.C. Chavantes and C.A.G. Pasqualucci, Correlation between near-infrared Raman spectroscopy and the histopathological analysis of atherosclerosis in human coronary arteries, Lasers Surg. Med. 30 (2002), 290-297.

[19] Operation Manual Holospec VPT System, Kaiser Optical Systems, Inc., Model HS- f/1.8i-NIR, Series HS- $0084,1994$.

[20] A.L. Boskey and R. Mendelsohn, Infrared spectroscopic characterization of mineralized tissues, Vibrat. Spectrosc. 38 (2005), 107-114.

[21] Y.N. Yeni, J. Yerramshetty, O. Akkus, C. Pechey and C.M. Les, Effect of fixation and embedding on Raman spectroscopic analysis of bone tissue, Calcif. Tissue Int. 78 (2006), 363-371. 
[22] H. Tsuda and J. Arends, Raman spectroscopy in dental research: a short review of recent studies, Adv. Dent. Res. 11 (1997), 539-547.

[23] S. Fukura, T. Mizukami, S. Odake and H. Kagi, Factors determining the stability, resolution, and precision of a conventional Raman spectrometer, Appl. Spectrosc. 60 (2006), 946-950.

[24] J.H. Lee, Y.M. Chang, Y. Han, H. Chung, S.H. Kim and S.B. Lee, Raman amplified-based long-distance remote, strain and temperature sensing system using an erbium-doped fiber and a fiber Bragg grating, Opt. Expr. 12 (2004), 3515-3520.

[25] M.A. Soto, G. Bolognini and F. Di Pasquale, Analysis of optical pulse coding in spontaneous Brillouin-based distributed temperature sensors, Opt. Expr. 16 (2008), 19097-19111.

[26] J. Ling and A.C. Bovik, Smoothing low-SNR molecular images via anisotropic median-difusion, IEEE Trans. Med. Imag. 21 (2002), 377-384.

[27] K. Toge, K. Hogari and T. Horiguchi, Performance prospects for distributed measurement of Raman gain characteristics in optical fibers, J. Lightwave Technol. 22 (2004), 1701-1706. 


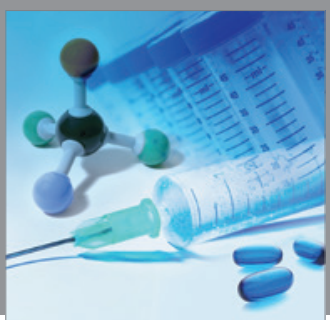

International Journal of

Medicinal Chemistry

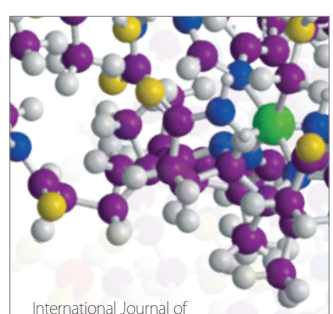

Carbohydrate Chemistry

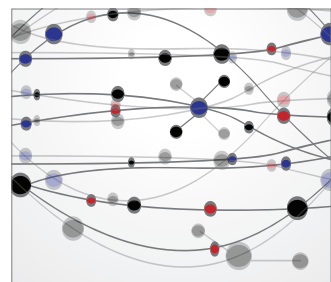

The Scientific World Journal
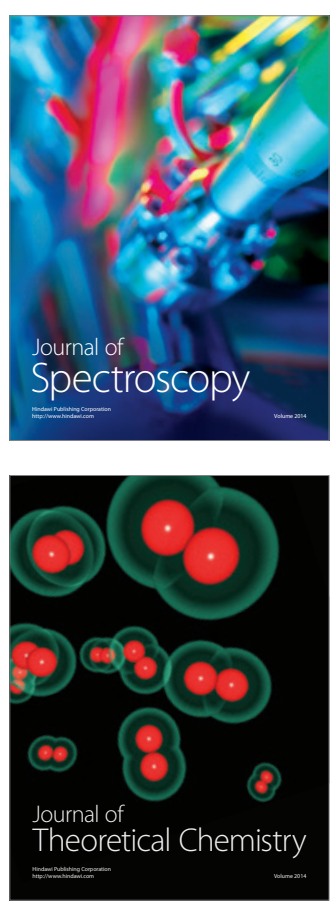
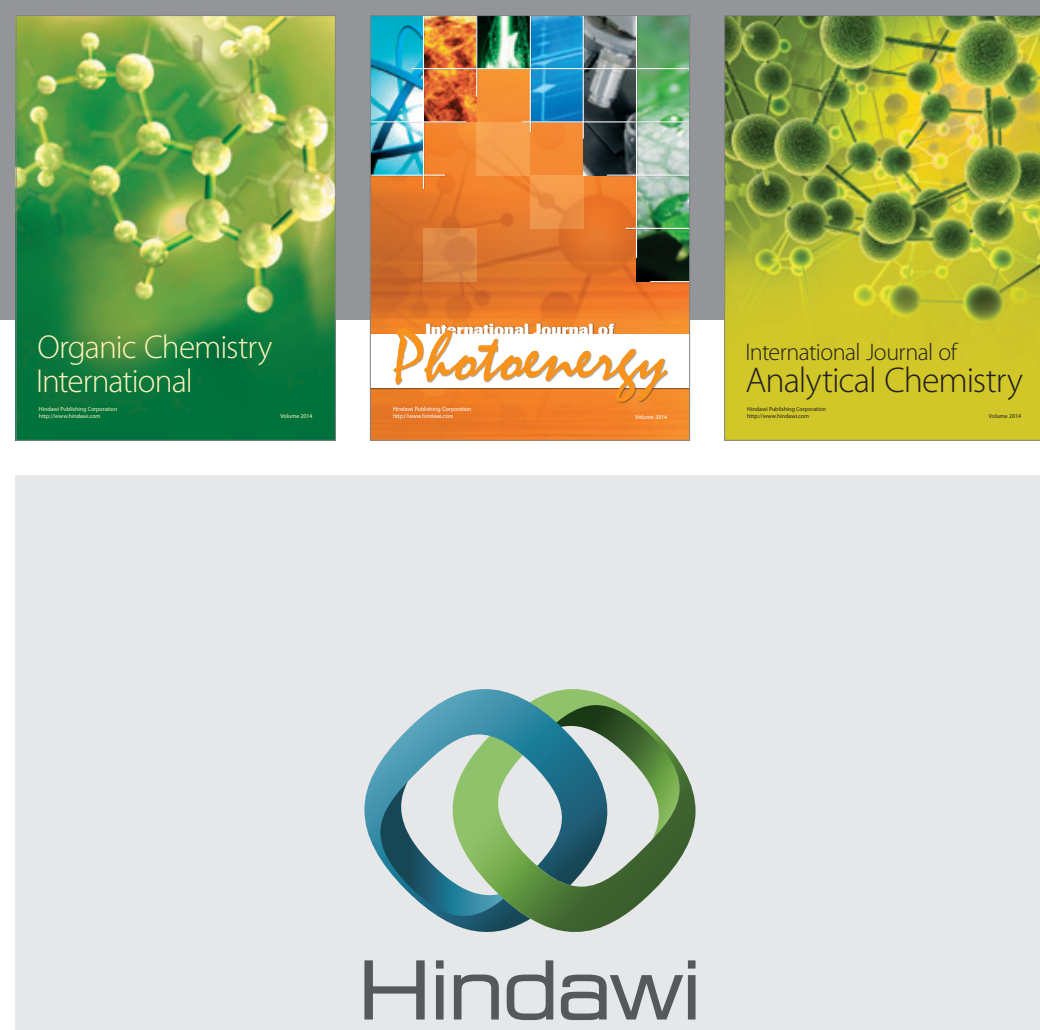

Submit your manuscripts at

http://www.hindawi.com
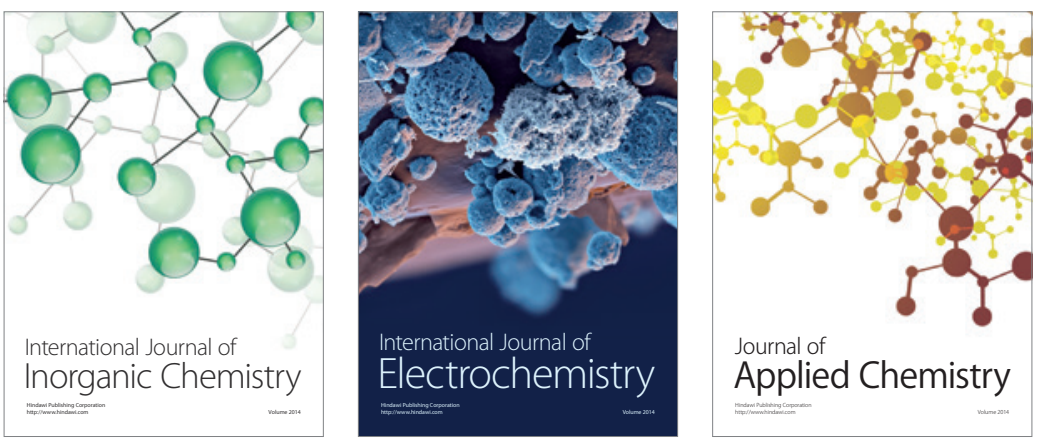

Journal of

Applied Chemistry
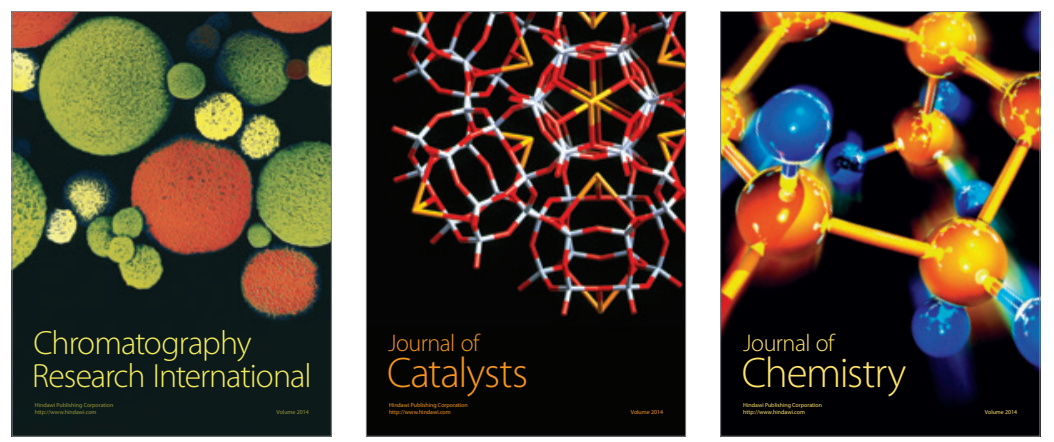
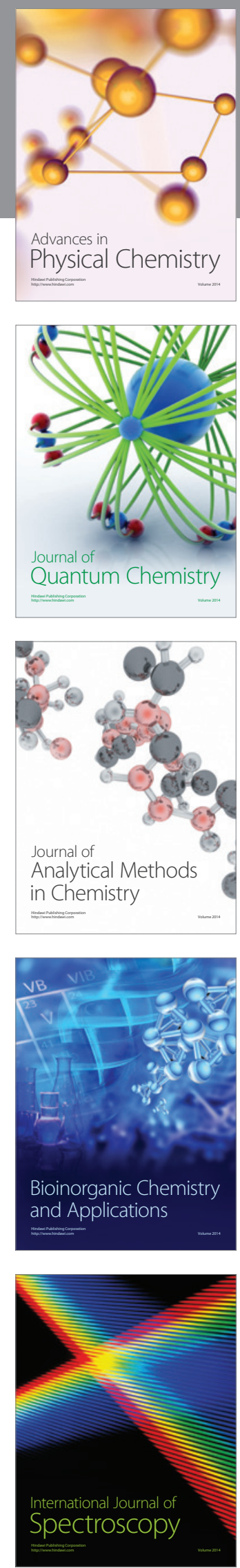